\title{
Advanced drug delivery systems: Nanotechnology of health design and its potential clinical Application
}

\author{
Mayurdhvajsinh R Sindha ${ }^{1 *}$, Jimishaben D Kher ${ }^{2}$ \\ ${ }^{1}$ Student, ${ }^{2}$ Assistant Professor, ${ }^{1}$ B N Patel Institute of Paramedical and Science, Anand, Gujarat, ${ }^{2}$ Parul Institute of Pharmacy \\ $\&$ Research, Faculty of Pharmacy, Parul University, Baroda, Gujarat, India \\ *Corresponding Author: Mayurdhvajsinh R Sindha \\ Email: jimmykher14@gmail.com
}

\begin{abstract}
Nanoparticles have been included in hundreds of identical types of products, and the novel properties of nanoparticles offers great promise to provide new technological approaches. However nanotechnology is an emerging technology which has prospective health and safety risks throughout its product life cycle. The nanoparticle plays a key role and it can conjugate with different drugs by various methods to deliver drugs to the target site. Clinical applications of different Nano systems in carcinoma therapy such as carbon nanotube, dendrimers, nanocrystal. The expansion in nanotechnology helps in the treatment of Parkinson's disease and alzheimer's diseases, Tuberculosis and in Ophthalmology are discussed in this article. Nano pharmaceuticals can be used to perceive diseases at much prior stages.
\end{abstract}

Keywords: Nano particles, Nanotechnology, Clinical application.

\section{Introduction}

\section{Nanotechnology}

Nanotechnology involves the scheme of matter on a near atomic scale to producestructures, devices, and materials. Nanoparticles are particles that have at least one dimension in the range of $1-100 \mathrm{~nm}$. At the Nano scale, fundamental mechanical, electrical, optical, and other properties can significantly differ from their bulk material counterparts. The new properties of nanomaterial offer significant promise toprovide new technological breakthroughs. Nanotechnology has been explored for creating lighter and stronger materials, forcleaning contaminated groundwater, for replacing toxic chemicalsin various applications, for increasing solar cell efficiency, and for selected cancer treatment. Nanotechnology is used in hundreds of products across different industries such as electronics, healthcare, chemicals, cosmetics, materials, and energy. Nanoparticles are taken up by cells more well organized than larger micro molecules and therefore, could be used as efficacious transport and delivery systems. For therapeutic applications, drugs can either be non-segregated in the matrix of the particle or attached to the particle surface. A drug targeting system should be able to control the fate of a drug entering the biological environment. Nanosystems with various compositions and biological properties have bee highly investigated for drug and gene delivery applications. An potent approach for achieving systemic drug delivery would be to rationally develop nano systems based on the understanding of their interactions with the biological environment, target cell population, target cell-surface receptors, changes in cell receptors that occur with progression of disease, mechanism and site of drug action, drug. Better understanding of the mechanism of uptake, intracellular trafficking, retention, and protection from degradation inside a cell are required for enhancing efficacy of the encapsulated therapeutic agent.

In recent years, proteinic and other drugs designed to target diffrent cellular processes have emerged, creating a demand for the development of drug delivery systems that can sense and respond directly to pathophysiological conditions. Micro- and nano-scale intelligent systems can maximize the potency of therapeutic treatments in numerous ways because they have the capacity to quickly detect and respond to disease states directly at the site, sparing physiologically healthy cells and tissues and thereby improving a patient's quality of life. This new class of "intelligent therapeutics" refers to intelligent and 
responsive delivery systems that are designed to perform diffrent functions like detection, isolation and/or release of therapeutic agents for the treatment of diseased conditions. To meet these requirements, researchers must be able to interface synthetic and hybrid materials with dynamic biological systems on the micro- and nano-length scale. Stimuli responsive biomaterials are very promising carriers for the development of therapeutics.

\section{Classification of Nano Materials}

Nano materials can be classified dimension wise into following categories:

1. Nano rods, nano wires have dimension less than $100 \mathrm{~nm}$.

2. Tubes, fibers, platelets have dimensions less than $100 \mathrm{~nm}$.

3. Particles, quantum dots, hollow spheres have 0 or 3 Dimensions $<100 \mathrm{~nm}$.

On the basis of phase composition, nano materials in various phases can be classified as,

1. The nano material is called single phase solids. Crystalline, amorphous particles.

2. Matrix composites, coated particles are included in multi-phase solids.

3. Multi-phase systems of nano material include colloids, aero gels, Ferro fluids, etc.

\section{Polymers in drug delivery systems}

Engineering polymeric nanostructures such as hyper branched polymers, dendrimers and polymeric micelles are a growing area of contemporary biomaterials science, due to their unique properties and large potential in drug delivery. For using polymers in drug delivery, a polymer must be biocompatible. Biocompatibility was defined by as the ability of a material to act with an appropriate host response in a particular application. Biocompatible polymers used in drug delivery are biodegradable with the formation of non harmful by products, such as non-toxic alcohols, acids and other easily eliminated low molecular weight products. They can indeed contribute to the drug release as a result of their erosion/degradation, in addition to drug diffusion through the polymeric material. Biodegradable polymer, in the development of drug delivery systems, must meet very specific requirements such as:

1. Biocompatibility backbone of the polymer and its degradation products.

2. Mechanical strength sufficient to meet the needs of individual applications.

3. Degradability with degradation kinetics matching a biological process.

4. Processibility using available equipment.

5. Solubility in different solvents.

6. Chemical, structural and application versatility.

7. Economically acceptable shelf life.

8. European Medicine Evaluation Agency (EMEA) or Food and Drug Administration (FDA), USA.

\section{Polymeric Micelles}

Polymeric micelles are nanoscopic (>100 nm) amphiphilic block copolymers with a core-shell structure (Fig. 1A). Polymeric nanoparticles nominated cores of biodegradable lipophilic polymers protected by an amphiphilic block copolymer that stabilizes their dispersion in aqueous media (Fig. 1B). Liposomes are vesicles consisting of one or more phospholipidic bilayer(s), with an aqueous core (Fig. 1C).

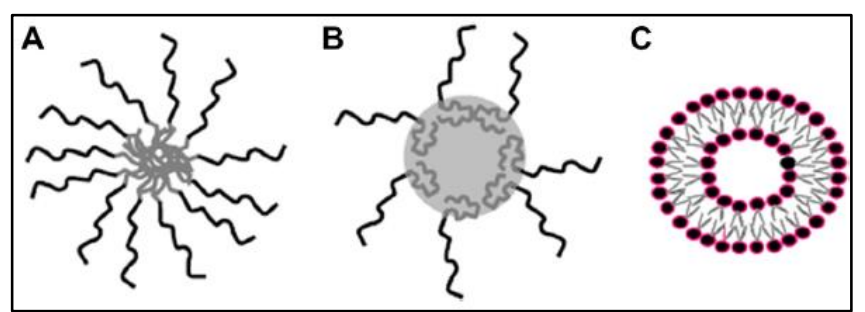

Fig. 1: Schematized polymeric Nano carriers: (A) micelle, (B) polymeric nanoparticle and (C) Liposome

In addition, polymeric micelles display larger cores than surfactant micelles, leading to more solubilization capacity than the regular micelles. Among the polymers displaying micelle-formation ability, micelles with blocks made of poly(ethylene oxide) are sterically stabilized and undergo low opsonization and uptake by the macrophages of the reticul endothelialsystem (RES), permiting the micelles to circulate bigger in blood polymeric micelles and nanoparticles have been investigated extensively for drug delivery. Polymeric micelles can be regarded as 
unique systems where aggregated lipophilic copolymers are in dynamic equilibrium with free unimers. While polymeric nanoparticles share a coreshell structure with micelles, they are matrix-type, solid-colloidal particles and, therefore exhibit generally more stability than micelles. They are larger $(100-500 \mathrm{~nm})$ than polymeric micelles $(10-100 \mathrm{~nm})$ and may display somewhat more polydisperse size distributions. Both polymeric micelles and nanoparticles are stabilized by surface-bound lipophobic polymers. Polysaccharides, such as chitosan, dextran and heparin, as well as poly (amino acids), have been used as corona-forming materials, in some cases for the delivery of taxanes.

Recently, the use of micelles prepared from amphiphilic copolymers for solubilization of poorly soluble drugs has attracted much attention. Amphiphilic block copolymers with having a large solubility difference between hydrophilic and lipophilic segments, have a tendency to self-assemble into micelles in a selective solvent. In an aqueous solution, micelles with core- shell structures are formed through the segregation of insoluble hydrophobic blocks into the core, which is surrounded by a shell composed of hydrophilic blocks. This coreshell structure facilitates their utilization, where depending upon the polarity the drug molecule can be entrapped in the

1. Core (non polar molecule)

2. Shell (polar molecule)

3. In-between the core and shell (intermediate polarity)

The unique characteristics of polymeric micelles, such as size in the nanometer range, high stability due to low critical association concentrations (CMC), and coreshell positioning, make them attractive for use in drug delivery systems in clinical applications, especially for lipophilic drugs with very low solubility in water the polymeric micelles.

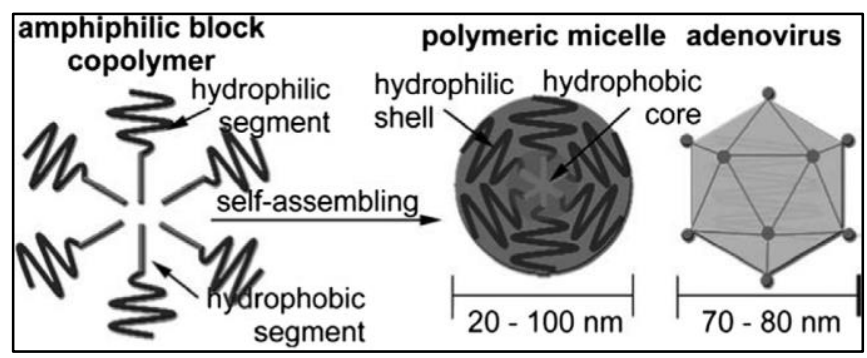

Fig. 2: Amphiphilic block copolymers and polymeric micelles

\section{Polymeric nanoparticles}

Polymeric nanoparticles (NPs) are $<1000 \mathrm{~nm}$ in size and are composed of bio stable polymers and copolymers. The drug molecules can be (i) encapsulated within the particle, (ii) adsorbed on the surface, or (iii) chemically linked to the surface of the particle. Polymeric nanoparticles possess a core-shell structure which can be varied by changing the composition of lipophilic and hydrophilic blocks on the polymer chains. The core consists of a dense polymer matrix in which a lipophilic drug can be encapsulated. The corona is made of a lipophoboic polymer, such as PEG, PVP, or polysaccharides, which serves to confer steric stability and stealth properties to the particles upon IV administration. Some of these structures are good candidates for drug delivery applications.

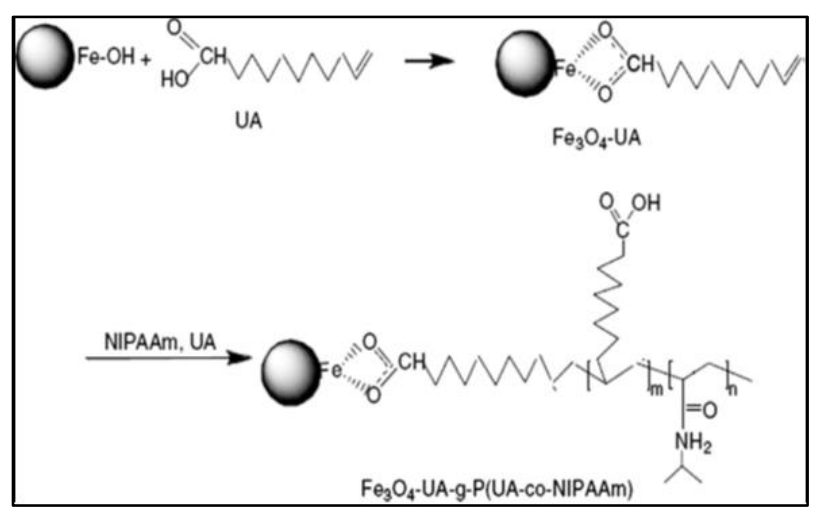

Fig. 3: The synthesis route of the Fe3O4-UA-g-P(UAco-NIPAAm)

\section{Dendrimers}

The term dendrimer, first proposed by To malia in 1985, was chosen due to its structural shape, with more branched, three-dimensional features that 
resemble the architecture of a tree. A dendrimer consists of three main structural components:

1. A focal core

2. (b)Building blocks with several interior layers composed of repeating units

3. Multiple peripheral functional groups.

\section{Carbon nanotubes in drug delivery}

Since the discovery of carbon nanotubes (CNTs) in 1991 CNTs have raised considerable due to excellent electrical, mechanical and surface properties that have made them unique candidates for a more range of clinical applications such as structural materials. Recently, its potential application in biotechnology has attracted much interest, as CNTs have be enreported to exhibit great advantages in biosensors, biomedical devices and drug delivery systems etc. Pristine, Moreover, some CNTs without any functionalization have been shown to be cytotoxic.

\section{Applications of Nanotechnology}

The different fields that find potential applications of nanotechnology are as follows:
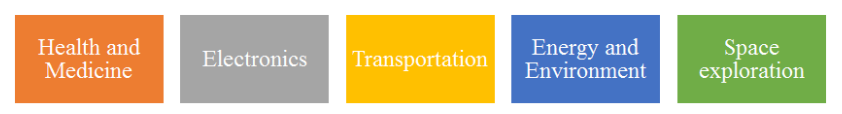

\section{Nanotechnology in health and medicine}

Different disease like diabetes, cancer, Parkinson's disease, Alzheimer's disease, cardiovascular diseases and multiple sclerosis as well as different kinds of serious inflammatory or infectious diseases (e.g. HIV) constitute a more number of serious and complex illnesses which are posing a major problem for the mankind. Nano-medicine is a clinical application of nanotechnology which works in the field of health and medicine. Nano-medicine constructs use of nano materials, and nano electronic biosensors.

With the help of nano medicine advance detection and prevention, improved diagnosis, actual treatment and follow-up of diseases is possible. Certain nano scale particles are used as tags and labels, biological can be performed quickly, the testing has become quickly sensitive and very flexible. Gene sequencing has become very efficient with the invention of nano devices like gold nano particles, these gold particles when tagged with small segments of DNA can be used for detection of genetic sequence in a sample.

Nano devices can be used in stem cell research in tracking and imaging them. It has its applications for science as well as translational medicine. Stem cells can be modulated by mixing of nano carriers with biological molecules. Nano devices can be used for intracellular access and for quick delivery and sensing of biomolecules. These technologies have a more impact in stem cell microenvironment and tissue engineering studies and have a unique potential for biomedical applications.

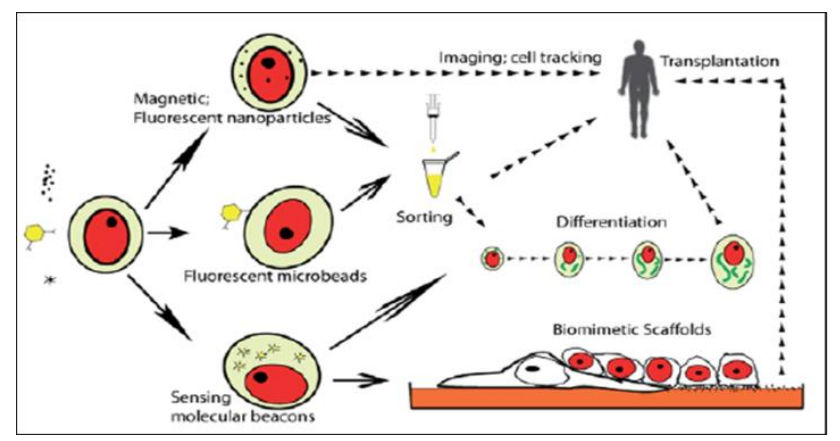

Fig. 4: Nanotechnology applications in stem cell biology and medicine.

\section{Nanotechnology, energy and environment}

Nanotechnology will play a censorious role in coming 50 years by protecting the environment and providing sufficient energy for a growing world. The advanced techniques of nanotechnology can help storage of energy, its conversion into other forms, ecofriendly manufacturing of materials and by superior enhanced renewable energy sources.

Nanotechnology is used on photovoltaic, for making them less expensive, light weight and very efficient, which can reduce the combustion of engine pollutants by nano porous filters, and can clean the exhaust mechanically, with the help of catalytic converters made up of nano scale noble metal particles and by catalytic coatings on cylinder walls and catalytic nanoparticles as additive for fuels.

\section{Medical use of Nano Materials}

Nano medicine is a relatively advanced field of science and technology. By interacting with biological molecules at nano scale, nanotechnology broadens the 
field of research and clinical application. Interactions of nano devices with bio molecules can be understood both in the extracellular medium and inside the human cells. Operation at nano scale allows exploitation of physical properties various from those observed at micro scale such as the volume/surface ratio.

Drug detoxification is another clinical application for nano medicine which has been used successfully in rats. Medical technologies can make use of smaller devices are reduced invasive and can possibly be implanted inside the body, and their biochemical reaction times are copious shorter. As compared to typical drug delivery nano devices are faster and further sensitive.

\section{Drug Delivery}

In nanotechnology nano particles are used for site distinct drug delivery. In this technique the required drug dose is used and side-effects are lowered importantly as the active agent is deposited in the morbid region. This more selective approach can lower costs and pain to the patients. Thus change of nano particles such as dendrimers, and nano porous materials find clinical application. Micelles obtained from block co-polymers, are used for drug encapsulation. They transport small drug molecules to the covet location. Similarly, nano electromechanical systems are utilized for the active release of drugs. Iron nano particles or gold shells are finding specific important application in the cancer treatment. A targeted medicine decrease the drug consumption and treatment expenses, making the treatment of patients cost effective.

Nano medicines used for drug delivery, are made up of nano scale particles or molecules which can better drug bioavailability. For maximizing bioavailability both at particular places in the body and over a period of time, molecular targeting is done by nano engineered devices such as nanorobots. The molecules are specific targeted and delivering of drugs is done with cell precision. In vivo imaging is different area where Nano tools and devises are being developed for in vivo imaging. Using nano particle images such as in ultrasound and MRI, nano particles are used as contrast. The nano engineered materials are being developed for more effectively treating illnesses and diseases such as cancer. With the advancement of nanotechnology, self-assembled biocompatible nano devices can be created which will detect the cancerous cells and automatically evaluate the disease, will cure and prepare reports.

The pharmacological and therapeutic properties of drugs can be improved by actual designing of drug delivery systems, by use of lipid and polymer based nano particles. The strength of drug delivery systems is their ability to improve the pharmacokinetics and bio-distribution of the drug. Nano particles are designed to circumvent the body's defense mechanisms can be used to improve drug delivery. New, complex drug delivery mechanisms are being developed, which can get drugs through cell membranes and into cell cytoplasm, thereby growing efficiency. Triggered response is one way for drug molecules to be used further efficiently. Drugs that are placed in the body can activate only on receiving a particular signal. A drug with less solubility will be replaced by a drug delivery system, having better solubility due to presence of both lip phobic and hydrophobic environments. Tissue damage by drug can be prevented with drug delivery, by regulated drug release. With drug delivery systems great clearance of drug from body can be reduced by changing the pharmacokinetics of the drug. Potential nano drugs will work by more specific and well-understood mechanisms; one of the major impacts of nanotechnology and nanoscience will be in leading development of completely new drugs with very useful behavior and less side effects.

\section{The applications of nano particles in drug delivery}

Abraxane, is albumin bound paclitaxel, a nano particle used for treatment of breast cancer and non-small- cell lung cancer (NSCLC). Nanotechnology can be efficiently used to treat different infectious diseases.

'Minicell' nano particle are used in untimely phase clinical trial for drug delivery for treatment of patients with advanced and untreatable cancer. The minicells are built from the membranes of mutant bacteria and were loaded with paclitaxel and coated with cetuximab, antibodies and used for treatment of a various of cancers. The tumor cells engulf the minicells. Once inside the tumor, the anti-cancer drug 
destroys the tumor cells. The larger size of minicells plays a superior profile in side-effects. The minicell drug delivery system uses less dose of drug and has less side-effects can be used to treat a number of various cancers with different anti-cancer drugs.

\section{Proteins and Peptide Delivery}

Protein and peptides are macromolecules and are called biopharmaceuticals. These have been identified for treatment of different diseases and disorders as they exert multiple biological actions in human body. Nano materials like nano particles and dendrimers are called as nano biopharmaceuticals, are used for targeted and/or controlled delivery.

\section{Cancer}

Due to the small size of nano particles can be of considerable use in oncology, particularly in imaging. Nano particles, such as quantum dots, with quantum confinement properties, such as size-tunable light emission, can be used in conjunction with magnetic resonance imaging, to produce exceptional images of tumor sites. As compared to organic dyes, nano particles are more brighter and need one light source for excitation. Thus the use of fluorescent quantum dots could produce a lofty contrast image and at a lower cost than organic dyes used as contrast media. But quantum dots are usually made of quite toxic elements

Nano particles have a extra property of high surface area to volume ratio, which allows diverse functional groups to get attached to a nano particle and thus bind to particular tumor cells. Furthermore, the 10 to $100 \mathrm{~nm}$ small size of nanoparticles, allows them to preferentially accumulate at tumor sites as tumors lack an effective lymphatic drainage system. Multifunctional nano particles can be manufactured that would detect, image, and then treat a tumor in future cancer treatment.

Nano wires are used to prepare sensor test chips, which can detect proteins and other biomarkers left behind by cancer cells, and detect and make diagnosis of cancer possible in the untimely stages from a single drops of a patient's blood.

Nano wires are used to prepare sensor test chips, which can detect proteins and other biomarkers left behind by cancer cells, and detect and make diagnosis of cancer possible in the untimely stages from a single drops of a patient's blood.

Nano technology based drug delivery is based upon three facts:

1. More efficient encapsulation of the drugs,

2. Successful delivery of said drugs to the targeted region of the body,

3. More successful release of that drug there.

Nano particles are used in cancer photodynamic therapy, wherein the particle is inserted within the tumor in the body and is illuminated with photo light from the outside. The particle absorbs light and if it is of metal, it will get heated due to energy from the light. More energy oxygen molecules are produced due to light which chemically react with and destroy tumors cell, without reacting with other body cells. Photodynamic therapy has gained importance as a noninvasive technique for dealing with tumors.

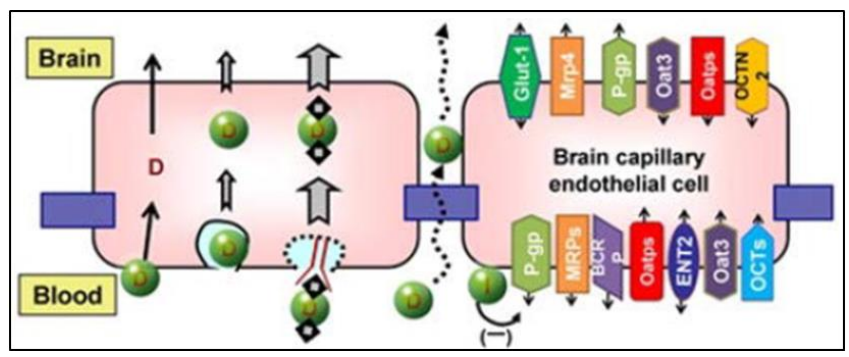

Fig. 5: Delivery of nano medicine to CNS through $\mathrm{BBB}$

\section{Nanotechnology in the treatment of neurodegenerative disorders}

One of the very important clinical applications of nanotechnology is in the treatment of neuro degenerative disorders. For the delivery of CNS therapeutics, various nano carriers such as, dendrimers, nano gels, nano emulsions, liposomes, polymeric nano particles, solid lipid nano particles, and nano suspensions have been studied. Transportation of these nano medicines has been moreveffected across various in vitro and in vivo $\mathrm{BBB}$ models by endocytosis and/or transcytosis, and early preclinical success for the management of CNS conditions such as, Alzheimer's disease, brain tumors, HIV encephalopathy and acute ischemic stroke has become possible. The nanomedicine can be advanced 
further by improving their BBB permeability and reducing their neurotoxicity.

\section{Parkinson's disease}

Aim of applied nanotechnology is regeneration and neuro protection of the central nervous system (CNS) and will more significantly benefit from basic nanotechnology research conducted in parallel with advances in neurophysiology, neuropathology and cell biology. The efforts are taken to develop potential technologies that directly or indirectly help in providing neuro protection and/or a permissive environment and active signaling cues for guided axon growth. In order to minimize the peripheral sideeffects of conventional forms of Parkinson's disease therapy, research is focused on the design, biometric simulation and optimization of an intracranial nanoenabled scaffold device (NESD) for the site-specific delivery of dopamine to the brain, as a strategy. Peptides and peptidicnano particles are newer tools for various CNS diseases.

Nanotechnology will play a key role in developing new diagnostic and therapeutic tools. Nanotechnology could provide devices to limit and reverse neuro pathological disease states, to support and promote functional regeneration of damaged neurons, to provide neuro protection and to facilitate the delivery of drugs and small molecules across the blood-brain barrier. For the delivery of CNS therapeutics, various nanocarriers such as dendrimers, nano gels, nano emulsions, liposomes, polymeric nano particles, solid lipid nano particles, and nano suspensions have been studied. Transportation of these nano models by endocytosis and/or transcytosis, and early preclinical success for the management of CNS conditions such as, Alzheimer's disease, brain tumors, HIV encephalopathy and acute ischemic stroke has become possible. Future development of CNS nanomedicines needs to focus on increasing their drug-trafficking performance and specificity for brain tissue using targeting moieties.

\section{Alzheimer's disease}

Alzheimer's disease (AD), which is the most common form dementia. Nano technology finds significant clinical applications in neurology. These approaches are based on the, early AD diagnosis and treatment is made possible by designing and engineering of a plethora of nanoparticulate entities with more specificity for brain capillary endothelial cells. Nano particles (NPs) have high affinity for the circulating amyloid- $\beta(\mathrm{A} \beta)$ forms and therefore may induce "sink effect" and improve the AD condition.

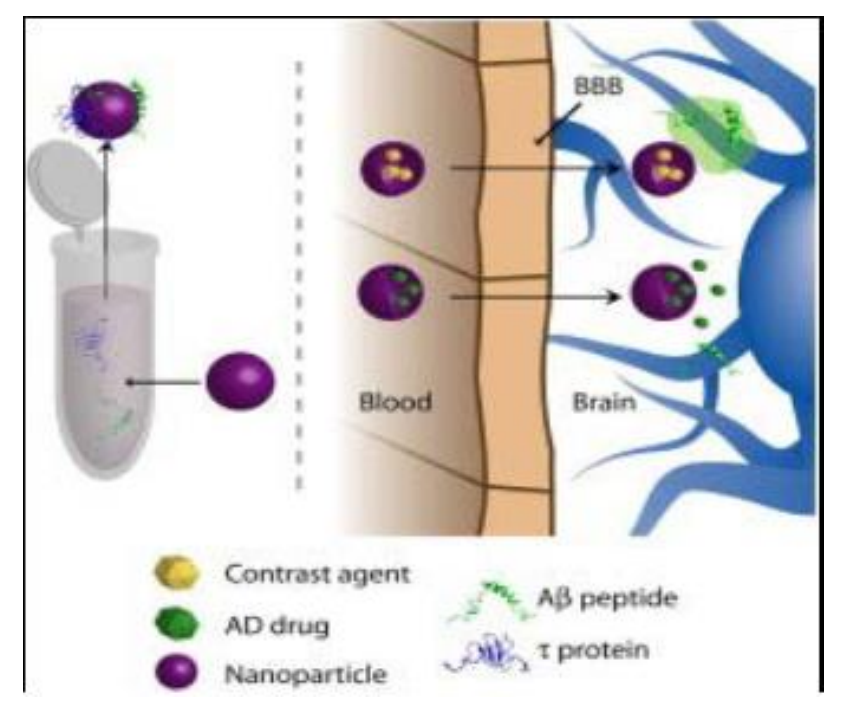

Fig. 6: Use of nano particles in Alzheimer's disease

\section{Tuberculosis treatment}

Tuberculosis (TB) is the deadly infectious disease. The long duration of the treatment and the pill burden can hamper patient lifestyle and result in the development of multi-drug-resistant (MDR) strains. Novel antibiotics can be designed to overcome drug resistance, cut short the duration of the treatment course and to reduce drug interactions with antiretroviral therapies. A nanotechnology is one of the most promising approaches for the development of further effective and compliant medicines. The advancements in nano-based drug delivery systems for encapsulation and release of anti-TB drugs can lead to development of an effective and affordable TB pharmacotherapy.

\section{The clinical application of nanotechnology in operative dentistry}

Nanotechnology aims at the creation and utilization of materials and devices at the atomic, and molecular level, supra molecular structures, and in the exploitation of special properties of particles of size 
$0.1 \mathrm{~nm}$ to $100 \mathrm{~nm}$. Nano filled composite resin materials are believed to offer excellent wear resistance, strength, and ultimate aesthetics due to their exceptional polishability and luster retention. In operative dentistry, nano fillers constitute spherical silicon dioxide ( $\mathrm{SiO} 2)$ particles with an average size of $5-40 \mathrm{~nm}$. The real innovation about nano fillers is the possibility of improving the load of inorganic phase. The effect of this high filler load is widely recorded in terms of mechanical properties. Micro hybrid composites with additional load of Nano fillers are the best choice in operative dentistry. The nano-filler used is aluminosilicate powder with a mean particle size of 80 ran 1:4 $\mathrm{M}$ ratio of alumina to silica and a refractive index of 1.508. These nano composites have superior hardness, flexural strength, modulus of elasticity, decreased polymerization shrinkage and also have excellent handling properties particle size of 80 ran 1:4 $\mathrm{M}$ ratio of alumina to silica and a refractive index of 1.508 .

\section{Applications in Ophthalmology}

The aim of nano medicine is the to monitor, control, construct, repair, defense, and improve human biological systems at the molecular level, with the help of nano devices and nanostructures that operate massively in parallel at the unit cell level, in order to reach medical benefit. Principles of nanotechnology are applied to nano medicine such as bio mimicry and pseudo intelligence. Some clinical applications of nanotechnology to ophthalmology are include treatment of oxidative stress; measurement of intraocular pressure; theragnostics; use of nano particles for treatment of choroidal new vessels, to prevent scars after glaucoma surgery, and for treatment of retinal degenerative disease using gene therapy; prosthetics; and regenerative nano medicine.

Recent research shows applications of different nanoparticulate systems like microemulsions, nanosuspensions, nanoparticles, liposomes, niosomes, dendrimers and cyclodextrins in the field of ocular drug delivery and also depicts how the various upcoming of nanotechnology like nanodiagnostics, nanoimaging and nanomedicine can be utilized to explore the frontiers of ocular drug delivery and therapy.

\section{Surgery}

In this technique, green liquid containing gold-coated nano shells is allowed to dribble along the seam and two sides are weld together. This method can be used arteries which have been cut during organ transplant. The flesh welder can be used to weld the artery perfectly.

\section{Tissue engineering}

In tissue engineering, nanotechnology can be applied to reproduce or repair damaged tissues. By using suitable nanomaterial-based scaffolds and growth factors, artificially stimulated cell proliferation, in organ transplants or artificial implants therapy nano technology can be useful, which can lead to life extension.

\section{Antibiotic resistance}

Antibiotic resistance can be decreased by use of nano particles in combination therapy. Zinc Oxide nano particles can decrease the antibiotic resistance and enhance the antibacterial activity of Ciprofloxacin against microorganism, by interfering with different proteins that are interacting in the antibiotic resistance or pharmacologic mechanisms of drugs.

\section{Immune response}

The nano device bucky balls have been used to alter the allergy/ immune response. They prevent mast cells from releasing histamine into the blood and tissues, as these bind to free radicals better than any anti-oxidant available, such as vitamin E.

\section{Nano pharmaceuticals}

Nano pharmaceuticals can be used to detect diseases at much earlier stages and the diagnostic applications could build upon conventional procedures using nanoparticles. Nano pharmaceuticals are an emerging field where the sizes of the drug particle or a therapeutic delivery system work at the nanoscale. Delivering the appropriate dose of a particular active agent to specific disease site still remains difficult in the pharmaceutical industry. Nano pharmaceuticals have enormous potential in addressing this failure of traditional therapeutics which offers site-specific targeting of active agents. Nano pharmaceuticals can 
reduce toxic systemic side effects thereby resulting in better patient compliance.

Pharmaceutical industry faces enormous pressure to deliver high-quality products to patients while maintaining profitability. Therefore pharmaceutical companies are using nanotechnology to enhance the drug formulation and drug target discovery. Nano pharmaceutical makes the drug discovery process cost effective, resulting in the improved Research and Development success rate, thereby reducing the time for both drug discovery and diagnostics.

\section{Conclusions}

Currently, there are many outstanding provocations that need to be labeled before product designers can fully apply these principles and make informed choices about nanoparticles. The potential of nanotechnology to engineer matter at the little scale is revolutionizing areas such as information technology cognitive science and biotechnology and is leading to new and interlinking these and other fields. By further research in nanotechnology, it can be useful for more aspect of human life. Medicine, regenerative medicine, stem cell research and nutraceuticals are among the leading sectors that will be modified by nanotechnology innovations.

\section{Source of Funding}

None.

\section{Conflict of Interest}

None.

\section{References}

1. Safari J, Zarnegar Z, "Advanced drug delivery systems: Nanotechnology of health design A review", J Saudi Chemical Soc 2014;18:85-99.

2. Suri SS, Fenniri H and Singh B, "Nanotechnology-based drug delivery systems", J Occupational Med Toxicol 2007:2-16.

3. Fonseca-Santos B, "Nanotechnology-based drug delivery systems for the treatment of Alzheimer's disease", Int J Nanomedicine 2015;10:4981-5003.

4. Nikalje AP, "Nanotechnology and its Applications in Medicine", Med Chem 2015:5-2.

5. Wanigasekara J, Witharana C, "Applications of Nanotechnology in Drug Delivery and Design - An Insight”, Curr Trends Biotechnol Pharm 10(1):78-91.

6. Gregory Morose, "The 5 principles of "Design for Safer Nanotechnology”, J Clean Prod 2009. doi:10.1016/j.jclepro.2009.10.001.

How to cite this article: Sindha MR, Kher JD. Advanced drug delivery systems: Nanotechnology of health design and its potential clinical Application. Int J Pharm Chem Anal 2019;6(4):95-103. 